\title{
Discovering the 21st Century Fountain of Youth: A Discussion of the Effectiveness of Popular Anti-Aging Therapies
}

\author{
Christopher J Salgado*, Charlotte E LaSenna, Rebecca Cissel, Xiaoyi Li, Catherine E Gordon, Andrea R Marcadis and Wrood Kassira \\ Division of Plastic and Reconstructive Surgery, DeWitt Daughtry Family Department of Surgery, University of Miami Miller School of Medicine, USA
}

*Corresponding author: Christopher J Salgado, Professor of Surgery, Division of Plastic and Reconstructive Surgery, DeWitt-Daughtry Family Department of Surgery, University of Miami Leonard Miller School of Medicine, Clinical Research Building (CRB)1120 NW 14th St, 4th Floor, Miami, Florida 33136, USA, Tel: 3052434500; Email: christophersalgado@med.miami.edu

Received date: February 22, 2014, Accepted date: April 25, 2014, published date: April 28, 2014

Copyright: ( 2014 Salgado CJ, et al. This is an open-access article distributed under the terms of the Creative Commons Attribution License, which permits unrestricted use, distribution, and reproduction in any medium, provided the original author and source are credited.

\section{Abstract}

Physical appearance has a significant effect on social and psychological aspects of self-esteem and overall wellbeing. With physical appearance so highly emphasized by current society, many are seeking ways to look younger and healthier. As a result, ant aging therapies are in demand and the market is constantly inundated with new products claiming to be the next best therapy for aging skin. Consumers as well as physicians often encounter difficulty in making informed decisions when choosing among treatment options.

Keywords: Anti-aging therapies; Skin aging

\section{Introduction}

A review of current literature can be a useful way to determine which therapies have undergone clinical testing and to assess and compare the outcomes of individual treatment modalities. During this process, critical assessment of the quality of studies is necessary in order to rule out studies with design flaws or excessive bias from corporate funders. Determining the level of evidence is also a useful tool in assessing the quality of a study. The Oxford Centre for Evidence-based Medicine [1] has developed a system of defining each level of evidence as follows: a systematic review with homogeneity of results reported from randomized, controlled trials (RCT) is classified as level 1a; an individual RCT with narrow confidence interval is classified as level $1 \mathrm{~b}$; all or none case series are classified as level 1c; a systematic review with homogeneity of cohort studies is classified as level 2a; an individual cohort study including low quality RCTs is classified as level $2 \mathrm{~b}$; "outcomes" research is classified as level 2c; a systematic review with homogeneity of case-control studies is classified as level 3a; individual case-control studies are classified as level 3b; case series are classified as level 4; and expert opinion without explicit critical appraisal or based on physiology or bench research is classified as level 5 . Results reported within this review have all been derived from level 1 and 2 studies.

In order to evaluate the effects of various treatment modalities on aging skin, it is important to understand the fundamental changes, which occur to the skin and subcutaneous tissues over time. Skin aging is a result of the combined effects of accumulated reactive oxygen species (ROS), telomere shortening, decreased hormone and cytokine levels, and a decrease in melanocytes and Langerhan's cells. Reactive oxygen species cause damage to lipid membranes, cellular enzymes, and DNA leading to cumulative tissue damage. Aging skin is characterized by atrophy, dryness, and laxity [2]. Intrinsic factors, such as programmed genetic changes, as well as extrinsic factors, such as sun exposure, and toxins including those from smoking, play a significant role in skin aging [3].
The primary focus of youth preservation is the face, as this is often our first impression of a person's age. Evidence of an aging face manifests as thinning skin, pigmentation changes, static wrinkle presentation with deepening facial folds, and sagging of the subcutaneous fat compartments. Epidermal thinning and loss of collagen leads to loss of skin elasticity [4]. Wrinkling results from a loss of facial fat combined with gravity and muscle pull. The aging face also undergoes many anatomical changes including descent of the brow and lower eyelid, changes in the contour of the upper eyelid, deflation of the infraorbital skin envelope, increased visibility of the lid-cheek junction and nasojugal crease, increased prominence and depth of the nasolabial fold, and development of marionette lines and jowls $[5,6]$. Boney remodeling also plays a role in the - aging face, with areas of the face undergoing specific and predictable resorption with aging. Specific areas of resorption include the superior and inferior alveolar bones, as well as supero-medial and infero-lateral orbit [7]. Twin studies have shown that smoking is associated with significant worsening of upper eyelid skin redundancy, lower lid bags, malar bags, nasolabial creases, jowls, upper lip lines, and lower lip vermillion lines than would be expected for one's chronological age, sun exposure, and genetics [8]. These superficial and anatomical changes are the targets of current facial rejuvenation therapies.

Clinical evaluation is the most frequently used tool to quantify the degree of age related changes before and after treatment, either inperson or via digital photography. Clinical scoring using validated assessment scales has proven to be highly reliable for the evaluation of the signs of facial aging including forehead lines, nasolabial folds, marionette lines, jawline, oral commissures, neck volume, glabellar lines, infraorbital hollow, upper cheek fullness, crow's feet, lip fullness, and lip wrinkles [9-12]. Though assessment scales have been used frequently to assess firmness, hyper pigmentation, fine lines, blotchy redness, tactile roughness, and clarity, they have yet to be validated. Alexiades Armenkdis developed and tested a scale along with formulas that have been widely used to quantify and calculate the percent improvement after treatment of individual parameters of skin aging, such as rhytides, laxity, dyschromia, elastosis, erythema-telangiectasia, texture, keratoses, and patient satisfaction [13]. 
In addition to clinical assessment scales, other assessment tools exist but are less frequently used. Skin surface topography using silicone impression-based grading is both a valid and reproducible measure of photo aging and dermal elastosis [14,15]. Biopsies may be used to histological assess increases in collagen and elastin deposition or epidermal thickness but are invasive and leave scars. Newer measurement technologies include the corneometer which measures hydration of the epidermis, cutometer which measures skin elasticity, and digital programs such as VISIA which combine multispectral imaging and complexion analysis software to quantify certain characteristics of the skin topography and subsurface such as UV damage, brown spots, fine lines, texture, pore size, and redness. This review will focus on the evidence behind various novel and traditional anti aging therapies that have been popularized in recent years with emphasis on those those are strongly proven to be effective.

\section{Sunscreen}

Exposure to UV radiation is a well-established factor in the acceleration of aging. Sunscreens block UV penetration of the skin and are an essential component of any anti-aging regimen. Though the benefit of sunscreen use for the prevention of skin aging has been demonstrated in animal models $[16,17]$, evidence of such benefits in humans has been lacking in the literature.

Recently, a randomized, controlled trial by Hughes et al. [18] tracked changes in skin aging in 903 adults aged 25-55 over for 4.5 years. Participants were randomly assigned to one of four different groups including: daily use of broad-spectrum sunscreen and $30 \mathrm{mg}$ of carotene, daily use of sunscreen and placebo, discretionary use of sunscreen and $30 \mathrm{mg}$ of carotene, and discretionary use of sunscreen and placebo. There were no differences in skin phonotype, sun exposure, pre-trial sunscreen use, or treatment compliance between groups. Upon completion of the trial, $77 \%$ of daily sunscreen users reported application of sunscreen at least 3-4 times per week versus $33 \%$ of discretionary users. Skin changes were assessed for differences pre-treatment and post-treatment using silicone-based replicas of the back of the hand in accordance with the Beagley and Gibson scale of microtopography. Results of the assessment showed no detectable increase in microtopography grade in the daily sunscreen group. These participants showed no progression of photo aging after 4 years of treatment. Skin aging advanced by $24 \%$ in the discretionary sunscreen group compared to the daily sunscreen use group (relative odds, 0.76 [ $95 \% \mathrm{Cl}, 0.59$ to 0.98$]$ ). There was no difference in microtopographic changes between groups assigned to carotene supplementation versus placebo. Because an increase by only one unit on the microtopography scale is associated with worsening of skin coarseness and wrinkling along with an increased risk of actinic keratoses and skin cancer $[19,20]$, the use of sunscreen was associated not only with cosmetic benefit but also with a reduction in the progression of malignant changes. Reported adverse effects of sunscreen are rare and mild including: contact allergy or skin irritation (3\%), greasiness (1\%), and interference with perspiration or stinging eyes after facial perspiration $(0.8 \%)$ [21]. Thus, daily sunscreen use is not only painless and inexpensive, but also the most effective intervention to lessen the continuation of photo aging and cutaneous malignancy caused by UV exposure.

\section{Antioxidants}

In recent years, antioxidants have been regarded as a panacea, but are the effects on skin supported by evidence? Free radicals cause damage to DNA, lipid membranes, and protein structures within cells, leading to photocarcinogenesis and photo-aging [22]. Free radicals or reactive oxygen species (ROS) also alter the structure of the skin by diminishing transforming growth factor-beta, which results in decreased collagen synthesis and increased elastin production [23-25]. Antioxidants have the ability to neutralize free radicals created in the skin due to UVA radiation exposure, toxins, and metabolic processes; and consequently protect the skin from aging and cancer. The body produces its own natural antioxidants, including superoxide dismutase, catalase, and glutathione (GSH) peroxidase. However, body stores are rapidly depleted when faced with excess UVA exposure and pollutants [22]. In addition, concentrations of antioxidants in the skin decrease with aging. Elderly subjects have $70 \%$ less alpha-tocopherol, Lascorbic acid, and total GSH than their younger counterparts [26].

Topical antioxidants have been developed in an effort to maintain antioxidant concentrations in the skin. There are many sources of antioxidants, among the most common are vitamins $\mathrm{C}, \mathrm{E}, \mathrm{A}$, selenium and tea polyphenols. These compounds can be combined for synergistic effects. For example, 15\% L-ascorbic acid and 1\% alpha tocopherol double the protection against UV induced erythema, sunburn, and thymine dimer formation versus ascorbic acid alone [27]. Due to their protective action within the skin, antioxidants are being increasingly incorporated into sunscreens and other skin care products. Unfortunately, antioxidants are unstable. In the case of vitamin $\mathrm{C}$, esterification is required to stabilize the compound and increase topical penetration. However, esterification also diminishes the antioxidant power of the product [28-30]. Therefore, improved topical delivery is often achieved at the expense of antioxidant capacity. Even though sunscreens with added antioxidants have proven to protect against the effects of photo-exposure to a greater extent than sunscreen alone $[31,32]$, this is not true of all products on the market.

Wang et al. [33] tested 13 popular commercial sunscreen products containing antioxidants according to 3 in vitro UVA labeling indices, including the new FDA proposed guidelines, and found that nearly all had either limited or no antioxidant effect. Despite product claims to include antioxidant protection, support is often lacking. In response to this issue, a trial by Palmer et al. [34] demonstrated the importance of quantifying antioxidant activity in order to produce an effective antiaging product. In order to ensure high levels of antioxidant activity in their product, ORAC testing was utilized. Total Oxygen Radical Absorbance Capacity (ORAC) is used by the U.S. Department of Agriculture to quantifying antioxidant capacity of both foods (ORACfn) and skincare products (ORACsc). While the average antioxidant skincare product averages an ORACsc score of 6,970 micromole TE/g, the regimen used in the trial scored within a range of 18,000-79,000 micromole TE/g for each of the various products. The regimen consisted of a facial wash, daily lotion, eye serum, and night crème, each including antioxidants in the form of coffee fruit extract and concentrated fruit and vegetable extracts. The control group regimen consisted of a control facial wash and a control moisturizer for day and night. Participants were selected based on mild-moderate facial photo damage and dryness and had to have used a moisturizer at least twice daily and a daily UVA/UVB facial sunscreen of SPF 30 or greater. Before the trial, participants underwent a 2-5 day washout period for all moisturizers, a 30 day washout period from all anti-aging products, a 6 month washout period for all prescription anti aging products, and a one year washout period for any cosmetic procedures. This 12-week, double-blind, randomized, controlled clinical trial enrolled 40 Caucasian females between the ages of 45-60 years, 
Fitzpatrick skin type I-IV, and Glogau score of II or III. Facial skin was evaluated before and after treatment based on clinical assessment using a 9-point scale including assessment of wrinkles, firmness, hyper pigmentation, blotchy redness, tactile roughness, and clarity. Clinical assessment was supported by digital photography, multispectral imaging and complexion analysis software (VISIA), silicone replicas of the crow's feet area, corneometer, cutometer, and participant selfassessment. Analysis revealed a significantly greater improvement in all parameters in the test group over the control group, including improvements in wrinkles (69.5\% vs $9 \%$ ), firmness ( $100 \%$ vs $81.8 \%$ ), hyper pigmentation $(78.2 \%$ vs $35.3 \%)$, blotchy redness $(82.6 \%$ vs $45.4 \%)$, tactile roughness ( $100 \%$ vs $81.8 \%)$, and clarity $(100 \%$ vs $81.8 \%)$. No adverse effects have been reported in the literature with the use of topical antioxidants. High antioxidant capacity products are an effective way to moderately reverse the various signs of photo aging. For best results, products that have been tested to ensure antioxidant activity are recommended.

\section{Retinoids}

Retinoids, vitamin A derivatives, have drastically improved the field of anti-aging skincare over the last several decades. Tretinoin, or alltrans-retinoic acid (RA), was the first topical prescription medication to demonstrate reversal and repair of both photo aging and intrinsic aging [35-38], and has been approved for this use since the 1990's [39]. RA has been extensively studied and consistently demonstrates significant improvements in fine facial wrinkles [40-42], mottled hyper pigmentation, and skin roughness [43-48] after 12 weeks of use, with further improvement after continued use [49-51]. RA interacts with nuclear receptors within the cell to influence epidermal proliferation and differentiation [52,53]. Through this mechanism, RA is able to counteract the thinning of the skin associated with aging by stimulating the synthesis and deposition of collagen and fibrillin. RA also diminishes the collagen degrading activity of matrix metalloproteinase's [53,54].

Retinol is the lipophilic precursor to RA and easily penetrates skin, where it is metabolized by keratinocytes. Retinol is very unstable in the presence of air and light, but recently, formulations have been developed to increase stability and to extend shelf life. Techniques include esterification with palmitate or acetate to create retinyl palmitate and retinyl acetate. Increased stability has greatly contributed to the formulation of OTC products, expanding the accessibility of this treatment. However, studies suggest that while retinol may be less irritating to the skin than RA, it is also less potent [55-57].

Until a 2012 study by Ho et al. [58], it was generally accepted that OTC retinol is not as effective as prescription retinoic acid. Ho et al. [58] conducted a randomized, double blind, controlled trial, comparing the effectiveness of non-prescription tri-retinol $1.1 \%$ vs. prescription Tretinoin $0.025 \%$ in 38 female participants aged $35-65$ with Fitzpatrick skin type I-IV and mild to moderate facial photo damage. The tri-retinol formula included retinol, retinyl palmitate, and retinyl acetate. Stability testing demonstrated maintenance of $95 \%$ of the initial concentration after 3 months. Clinical assessment on a nine-point scale with half-point increments was used to evaluate participants for fine wrinkles, coarse wrinkles, skin firmness, skin tone/brightness, mottled pigmentation, tactile roughness, and global photo damage improvement. In all parameters, there was no significant difference between the two treatments. Thus, the authors concluded that it is possible to formulate an OTC retinoid product that is as effective as prescription RA. It is important to note that the lowest available strength of RA (0.025\%) was selected for comparison to the tri-retinol formulation. Efficacies of higher concentration RA might yield different results. Adverse effects of retinoid use include overall mild erythema, dryness, scaling, peeling, burning, stinging, itching, and tightness. The use of moisturizer can help diminish these effects. Retinoids also increase photosensitivity, requiring the use of daily sunscreen. All adverse effects were rated as mild in both the triretinol and RA groups. The tri-retinol group maintained erythema throughout the course of treatment, while the RA group peaked and diminished after 4 weeks. Retinoids appear to be a universally effective treatment choice for all skin types and levels of photo damage. Because $\mathrm{RA}$ is also an effective treatment for acne, it is a sensible treatment choice in patients who suffer from acne and also demonstrate signs of photo damage. Over-the counter retinoid products should be selected carefully due to variations in stability and effectiveness among different formulations.

\section{Chemical Peels}

Another common minimally invasive procedure to fight the signs of aging is chemical peels. Chemical peels consist of controlled destruction of the epidermis with or without the dermis in order to remove superficial lesions and allow for regeneration of tissue to correct defects. Chemical peels are ideal for patients with wrinkling, loss of elasticity, as well as irregular pigmentation and actinic keratosis from photo damage. These patients must be distinguished from patients with gravitational effects of aging and volume loss who require surgical correction. However, chemical peels are a good supplement to the surgical options to give a more youthful surface appearance. The ideal chemical peel patient has fair skin with mild, shallow wrinkles. Relative contraindications to chemical peels include cutaneous radiation history, smoking, active or frequent HSV infections, diabetes, and tendency for hypertrophic or keloid scars. Elevated levels of estrogen, such as in pregnancy or with the use of OCPs, and photosensitizing drugs cause hyper pigmentation and should also be avoided. Patients are recommended to avoid pregnancy during or within the first 6 months of a peel. Concurrent or recent isotretinoin use is an absolute contraindication $[59,60]$.

Chemical peels are categorized according to peel depth: superficial, medium, and deep. Superficial peels, using alpha hydroxy acids and beta hydroxyl acids or Jessner's solution (14 g salicylic acid, $14 \mathrm{~g}$ lactic acid, $14 \mathrm{~g}$ resorcinol in $100 \mathrm{cc}$ ethanol), result in epidermal sloughing and an inflammatory response in the upper papillary dermis while stimulating collagen production. This is reserved for patients with mild photo damage, superficial lentigines, and fine wrinkles. Medium peels, using Jessner's peel or $\mathrm{CO}_{2}$ and $35 \%$ trichloroacetic acid (TCA) solution, slough the epidermis and cause variable amount of necrosis and inflammation in the papillary dermis. Medium peels are reserved for patients with epidermal growth, moderate photo damage, and mild to moderate wrinkles or acne scars. These peels avoid inflammation in the reticular dermis, minimizing the adverse effects of deep peels. Deep peels, with increased concentration of TCA solution or phenol, slough the epidermis and papillary dermis and cause inflammation of the reticular dermis. These are reserved for deep wrinkles but increase the risk of scarring and hypo pigmentation $[61,62]$.

Various chemicals have been used as superficial peeling agents, most commonly being alpha-hydroxy acids, such as glycolic acid, or beta-hydroxy acids, such as salicylic acid. A recent study compared the efficacy of two types of superficial chemical peels, glycolic acid (GA) 
and capryloyl salicylic acid (LHA), a novel derivative of salicylic acid. Volunteers were randomly assigned to treatment of one side of the face with LHA vs. GA in increasing concentrations as tolerated and then compared. At 12 week follow up, $41 \%$ of LHA-treated and $30 \%$ of GA-treated subjects demonstrated significant reduction of fine lines/ wrinkles. Also notable, $46 \%$ of LHA subjects and $34 \%$ of the GA subject's demonstrated reduction in hyper pigmentation compared to baseline. The study concluded the 5-10\% LHA peel to be as effective as $20-50 \%$ GA peel in reducing fine lines/wrinkles and hyper pigmentation suggesting LHA to be a viable alternative [61].

Langsdon et al. [62] recommend pre-peel preparation with exfoliation, hydration, and sun protection. Patients are recommended to use sunscreen with SPF 30 or higher and a facial exfoliant cream containing glycolic acid for at least 2 weeks before the procedure. Tretinoin can be used in patients with thick, oily skin and hydroquinone can be used in patients with significant hyper pigmentation or melasma. Authors also recommend increasing TCA doses between sessions from $15 \%$ to $35 \%$ for best wrinkle and photo damage reduction [62].

Another study recommends the use of glycolic acid combined with $35 \%$ TCA in medium depth peeling. Thirteen patients underwent treatment with $70 \%$ GA plus $35 \%$ TCA to one side of the face and Jessner's solution plus 35\% TCA to the other side of the face. The GATCA peel was more effective at reducing actinic keratosis than the JSTCA peel. The JS-TCA peel resulted in a stronger inflammatory response with more neovascularization and fibrosis while the GATCA peel resulted in more neoelastogenesis. The GA-TCA peel may also be more effective in correcting damaged elastic fibers. Authors concluded that the GA-TCA combination is an effective option for medium depth chemical peeling in treating photo damaged skin [63].

Concern has been raised regarding the carcinogenicity of trichloroethylene (TCE) and its oxidative metabolite, trichloroacetic acid (TCA). One study examined chronic inhalation assays and oral gavage in mice and implicated TCA as a cancer-causing compound, independent of TCE exposure [64]. Another study demonstrated cytotoxic and genotoxic effects of TCA on human lymphocytes in vitro [65]. The National Toxicology Program (NTP) has classified TCA as "reasonably anticipated to be a human carcinogen" based on these and similar studies [66]. As TCE is used as an industrial solvent, people in professions involving years of exposure to this chemical have been shown to develop liver cancer, kidney cancer, and non-Hodgkin's lymphoma; however, these reports are based on small pools of subjects and are confounded by the presence of other solvents and carcinogenic risk factors in the workplace [67]. We are unsure of the actual potential of cancer caused by TCA peeling as there are no human in vivo studies assessing this risk. Chemical peel procedures involve exposure to TCA for only a short period of time and no evidence of carcinogenesis following this limited exposure has ever been documented.

\section{Neuromodulators (BotoxTM, DysportTM, XeominTM)}

Botox (botulinum neurotoxin) treatment has been a mainstay in non-surgical facial rejuvenation for over 20 years. There are several forms of botulinum neurotoxin, but only botulinum neurotoxin type A (BotoxTM, DysportTM, XeominTM) is approved in the US for cosmetic purposes [68]. While Botox is officially indicated for the temporary improvement of moderate to severe glabellar lines in adults
65 and younger, it is widely used off-label for other anti-aging purposes including correction of horizontal forehead lines and crow's feet [69].

One of the major advantages of neuromodulators is the ability to individualize each patient's treatment plan by altering both the dosage and the treatment areas according to each patient's needs. Furthermore, treatment plans are often combined with other antiaging therapies including retinoids, lasers, and fillers as needed. Botox has been shown to be safe, not only in the short term, but even after repeated treatments [68]. In a meta-analysis of 36 studies involving 2309 subjects, no serious adverse effects were reported and the level and duration of efficacy was maintained over repeated rounds of injections with no major safety concerns [69].

The most frequent adverse effects following Botox treatment (reported by $>5 \%$ of patients in clinical trials) are headache, respiratory tract infection, blepharoptosis, back pain, acne [69] and bruising [68]. These effects are minor and can generally be managed with simple interventions [68]. In addition, the rates of ptosis generally decrease with increased clinician experience [69]. In 2009, a black box warning was issued for BoNT-A products related to diffusion of the toxin beyond the treatment site. However, this effect is rare and is usually related to non-aesthetic uses of Botox [68]. Another rare complication of Botox treatment is the formation of neutralizing antibodies against a particular form of botulinum neurotoxin. In this case, the Botox treatments decrease in their efficacy after repeated use. Switching to a different formulation of botulinum neurotoxin (such as BoNT-B) restores the efficacy of the treatments. The effect of neutralizing antibodies could become more problematic in the future with the aesthetic market shifting towards a younger population of recipients who receive the treatments for a greater number of years [68].

\section{Fat Grafting and Stem Cell Transplants}

Volume loss has been recognized as a vital, if not primary, cause of facial aging [70]. The realization of the contribution of volume loss to facial aging initiated the "conservation of tissue" paradigm of facial rejuvenation 15 years ago. Maintaining the fat and skin volume of the face is not only important for maintaining a youthful looking appearance, but also for avoiding the "operated on" look in patients who have undergone facial surgery [71]. The increased demand for minimally invasive procedures with minimal downtime, as well as the ease of obtaining autologous adipose tissue has contributed to the emergence of fat transplantation as a mode of anti-aging therapy [72].

Autologous fat grafting, sometimes referred to as fat transfer, lipoinjection, or liposculpture, is performed by aspirating fat tissue from one area of the body and injecting it into another. There are many cosmetic applications, including lipoatrophy or asymmetry of the face, lip and facial augmentation, anti-wrinkle therapy [73]. And reconstructive use in patients with scars, radiation damage, and chronic ulcerations [74]. For anti-aging purposes, it is often injected into the periorbital region, cheeks, nasolabial folds, and perioral region [71]. Experimental evidence has shown that fat grafting increases volume in the face by a combination of neovascularization and survival of transplanted pre-adipocytes and adipocytes as well as fibrofatty tissue replacement due to inflammation created in the transplantation process [72].

Autologous fat has many properties of the "ideal filler." It is safe (non-immunogenic, non-carcinogenic, non-teratogenic), effective 
(looks and feels natural, reproducible long term benefit), and practical (cost effective and easy to use) [74]. It is also durable and one harvest can give an abundance of transferrable fat that can be frozen and stored for 1 year [71]. This storing of adipose allows for "touch up" procedures if necessary, albeit at a lower long-term survival than "fresh" fat. Also, in addition to augmenting facial volume, fat grafting has also been reported to improve the quality and texture of the aging skin [74], which has been attributed to the significant stem cell population in the transferred fat [70].

The two major potential problems in fat grafting are variable resorption of adipose over time, and the potential for contour deformities [70]. Other complications include edema and bruising, which rarely last greater than 2 months. More severe, yet rare complications include graft necrosis, infection, and damage to underlying structures, blindness, and stroke. It often takes 3-6 transfers for optimal augmentation. It is important to avoid overcorrection in one session, because fat survival is better when overall amounts are small $(1-3 \mathrm{cc})$ in each area and minute deposits (0.1cc) are implanted [72].

A prospective longitudinal study by Guijarro-Martinez et al. [74] used photographs to measure the efficacy of fat grafting. Photographic documentation was obtained for 9 patients pre-procedurally and at 3, 6, 12, and 24 months post-procedure. Patient satisfaction was evaluated using a visual analogue scale (VAS) ranging from 1-10 at 18 months post-op. High patient satisfaction was reported (8.7 average VAS). Retrospective analysis of the photo documentation showed progressive volumetric decrease for up to 6 months post procedure. Volume seemed to stabilize at about 3-4 months, but a subtle decrease sometimes occurred for up to 1 year. Beyond that, volume was demonstrated to remain constant for 8-12 years [74]. Another prospective study by Fontdevila et al. [75] evaluated the long term viability of malar fat grafting using CT scans to volumetrically measure fat tissue. This study found that there was a statistically significant improvement in facial fat volume up to 12 months post procedure, and that it actually increased from the 2 months to the 12 months post procedure follow up. The contrasting results from these studies and the absence of clear, consistent, objective evidence of the survival of fat grafts is the reason why many plastic surgeons choose to use other fillers for facial volume replacement. However, the most experienced plastic surgeons have reported clinical results showing both short and long term persistence of transferred grafts and promote autologous fat as the ideal soft tissue filler [75].

Adipose derived stem cells (ADSC) can be isolated from the lipoaspirate used for fat grafting and are now being injected as an addition to the autologous fat graft [76]. While the injection of additional ADCs in fat grafts still requires IRB approval, this technique has already shown many benefits, including enhanced angiogenesis, improved graft survival, and reduction in graft atrophy [73]. The mechanism of the stem cells' graft enhancement has not been completely elucidated, but they are thought to work by contributing to neo-angiogenesis in graft tissues. Stem cells act as endothelial progenitor cells or angiogenic factor releasing cells and are known to secrete angiogenic factors such as VEGF and hepatocyte growth factor. In vitro, they demonstrate the ability to proliferate in response to a hypoxic insult remote from their resident niche. Additionally, further in vitro studies show increasing ADSC proliferation with greater degrees of hypoxia [76].

There are several advantages to using adipose derived stem cells in fat grafts. They are an easily attainable source of adult stem cells
(1:100-1:1500 cells) compared to other tissues such as bone marrow, where the percentage of stem cells is not as high. The additional cost per procedure of using stem cells in the fat graft is negated by the fact that fewer touchup procedures have to be performed on those patients postoperatively than they do for the fat grafts without stem cells [76]. A prospective trial by Sterodimas et al. [76] compared fat grafting with and without the addition of adipose derived stem cells.

Twenty patients with congenital or acquired facial defects were randomly divided into 2 groups: 10 treated with autologous fat transplantation: $12-165 \mathrm{~mL}$ per session (group A), and 10 were treated with ADSC enriched lipografts: $8-155 \mathrm{~mL}$ per session (group B). Patients were followed at 6,12 , and 18 months post treatment and were asked to rate their overall satisfaction with their post treatment facial appearance on a validated [72] 5 point scale (1=poor, $2=$ fair, $3=$ good, $4=$ very good, $5=$ =xcellent). In group A, 3 patients achieved aesthetically acceptable results after a single treatment, but the remaining 7 required additional sessions of autologous fat grafting ( 3 had one additional treatment, 4 needed 2 additional treatments). All of the patients in group B required a single treatment session. Analysis of patient satisfaction clearly demonstrated better results in group B, maintained until 18 months. However by 18 months, there was no statistical difference between the groups in terms of patient satisfaction [76]. This study demonstrates that the addition of ADCs to fat grafts allows for fewer treatment sessions and improved cosmesis between sessions.

\section{Ablative Fractionated Photothermolysis (AFP)}

Laser resurfacing began with ablative $\mathrm{CO}_{2}$ lasers, which rapidly advanced to become the standard for non-operative wrinkle reduction. However, ablative lasers not only required 2-3 weeks for reepithelialization, resulting in 3-4 months of postoperative erythema, but also carried significant risks of scar and dyspigmentation. In response to these cosmetic issues, non-ablative lasers were developed in an attempt to similarly augment collagen formation and tighten tissue without disrupting the layers of the skin. Unfortunately, with improved safety came reduced efficacy. It was not until 2008 with the release of an ablative fractionated laser (AFP) that the most effective results could be produced with less risk.

Unlike the original ablative lasers, which fully remove the top surface of the skin, fractionated lasers create closely spaced, but spatially distinct micro-ablations that are able to safely treat greater depths within the dermis. Additionally, the presence of surrounding areas of unaffected dermal and epidermal tissue between ablations allows for enhanced wound healing and tissue contraction, resulting in a more rapid recovery, greater efficacy of treatment, and reduced occurrence of adverse effects such as scarring and dyspigmentation.

AFP is effective for the reversal of photo damage, pigmentation, collagen breakdown, and laxity. AFP also produces greater improvements in skin tightening and texture compared to NAFP. Many types of fractionated laser treatment modalities currently exist, including erbium-doped yttrium aluminum garnet (Er:YAG) and yttrium scandium gallium garnet (YSGG). A study by LaTowsky and colleagues showed statistically similar effectiveness amongst four fractional laser devices up to 6 months post treatment, including Active and Deep $\left(\mathrm{CO}_{2}\right)$, Fraxel Repair $\left(\mathrm{CO}_{2}\right)$, Quadralase $\left(\mathrm{CO}_{2}\right)$, and Pearl (YSGG) $[77,78]$. Fraxel Repair pain scores ranged higher than other devices and resulted in the most pinpoint bleeding, but was the most time efficient device and produced the highest patient 
satisfaction at 1 month post treatment; Active and Deep also caused pinpoint bleeding, but patients scored it highest for ease of healing and willingness to repeat treatment [79].

In order to quantify the effects of ablative fractionated phototherapy, Tierney et al. [78] followed 45 patients (39 women) aged 45-75 of Fitzpatrick skin types I-III throughout 2-3 sessions (mean 2.4) of treatments. Patients experienced pinpoint bleeding up to 48 hours post-treatment; edema, crusting, and severe erythema up to 72 hours post treatment; and moderate erythema for up to one week post treatment. However, there were no cases of scarring, postinflammatory dyspigmentation or infection. Blinded physician photographic assessments occurred at 6 months post-treatment, based on a modified scale by Alexiades-Armenakas [79]. Treatment resulted in $48.5 \%$ mean improvement in skin texture, $50.3 \%$ mean improvement in skin laxity, $53.9 \%$ mean improvement in dyspigmentation, and 95\% mean improvement in overall cosmetic outcome. The authors credited AFP with significantly greater improvements than those seen with NAFP devices. NAFP has lasting effects with $74 \%$ of improvements maintained at 2 years post treatment [80]. Additionally, ablative fractional phototherapy has been proven to be safe and effective for tightening of skin in the neck [81] and for the treatment of photo aged peri-ocular skin, including areas within the orbital rim [82].

\section{Photodynamic Therapy}

For patients who are not ready for aggressive treatments such as ablative skin rejuvenation, photodynamic therapy (PDT) is a milder option with results that are still more effective than non-ablative rejuvenation methods. PDT was originally approved to treat actinic keratosis, but off-label uses include treatment of malignant lesions of the skin such as superficial basal cell carcinoma and Bowen's disease. Following treatment, clinicians also noted reversal of the signs of photo aging including improvement of dyspigmentation, skin texture, fine lines, and overall complexion [83]. Since this discovery, PDT has been further developed for the purpose of photo rejuvenation.

PDT begins with topical application of a photosensitizer. The photosensitizer is absorbed and concentrated within premalignant and malignant cells to a greater extent than, non-damaged cells. A light source is used to activate the photosensitizer, creating reactive oxygen species (ROS) that selectively destroy the cells with higher photosensitizer concentration [84]. While common light sources include intense pulsed light (IPL), light emitting diode (LED), and lasers, non-laser light sources can be used to cover large surface areas, such as the face, in less time. The ROS created by this method have a short life span and therefore have a low mutagenic potential for extensive or systemic DNA damage [85]. Two photosensitizers, 5aminolevulinic acid (ALA) and methyl aminolevulinate (MAL), are approved for dermatological use. Of note, the IPL-ALA combination has proven to be safe and effective for use on darker skin-types (Fitzpatrick III-IV) [86]. Compared to IPL alone, Dover et al. [87] found ALA-IPL treatment to have significantly greater improvements in global score for photo aging ( $80 \%$ vs $45 \%$; $\mathrm{P}=0.008)$, fine lines $(60 \%$ vs $25 \% ; \mathrm{P}=0.008)$, and mottled pigmentation $(85 \%$ vs $20 \%$; $\mathrm{P}<0.001)$; however, this combination was also associated with more adverse effects such as erythema (50\% vs $15 \%$ ), scaling and dryness (50\% vs $15 \% ; \mathrm{P}=0.04)$, edema ( $50 \%$ vs $10 \% ; \mathrm{P}=0.04)$, and crusting with vesiculation $(20 \%$ vs $5 \% ; \mathrm{P}=0.11)$. A histologic and morphometric study by Issa et al. [88] demonstrated a significant increase in collagen and decrease in solar elastosis at 3 months with continued improvements 6 months post-treatment with red light and MAL.

There is no standardized protocol for PDT for photo rejuvenation as far as optimal incubation time, photosensitizer concentration, light dose, and number of sessions. The varied results reflect user dependent technique for application and the paucity of literature on the subject. In a review of the existing literature by Karrer et al. [89], PDT was upheld as an effective method for the reversal of fine lines and reduction of tactile skin roughness along with improvement of skin smoothness, actinic elastosis, skin color, and hyper pigmentation. PDT has less effect on coarse wrinkles and sebaceous gland hyperplasia; however, PDT with IPL is especially effective for the removal of telangiectasias and improvement of mottled hyper pigmentation. Another benefit of PDT is that adverse effects are generally mild to moderate, with erythema reported most often and occasional reports of crusting or mild superficial peeling. Although results are seen after one session, most studies are designed to include 3 sessions for maximal results. PDT is an advantageous form of photo rejuvenation due to its ability to affect multiple parameters of skin aging while also targeting $\mathrm{AK}$ and other dysplastic cells.

\section{Combination AFT and PDT}

As successful anti-aging therapies are discovered, combinations of therapies undergo evaluation for synergistic effects. Recently, a study by Haedersdal et al. [90] tested the effect of fractional $\mathrm{CO}_{2}$ laser pretreatment on uptake of the photosensitizing agent MAL for PDT. AFT was used in Yorkshire swines to create tunnels through which MAL could penetrate deeper into the dermis and elicit a full thickness response from subsequent PDT. Results confirmed the hypothesis of deeper penetration with significantly greater levels of PDT activity, i.e. PpIX fluorescence and PpIX photo bleaching, extending into the deep dermis and subcutaneous fat compared to superficial extension with MAL-PDT alone. They concluded that penetration of photosensitizer is the main limiting factor in PDT, not penetration of the light source. Adverse effects included slight to intense erythema and pronounced inflammatory response. This combination is in the early phases of testing, but offers a promising possibility for boosting the effectiveness of PDT in future clinical practice.

\section{Surgery}

Many patients elect to undergo aesthetic facial surgery in an effort to reverse signs of aging. Options include rhytidectomy, upper face reconstruction with or without blepharoplasty/brow lift/forehead lift/ orbital suspension, perioral reconstruction, and neck reconstruction. Most surgical reconstruction is combined with fat grafting, fractionated lasers, fillers, and or botulinum toxin treatment. It is important for patients undergoing any procedure to understand that the skin progressively tightens over time, requiring 6-12 months to see full results. Aesthetic facial surgery is typically a rewarding experience with good results. A recent study assessed perceived ages of patients before and 6 months after surgery. Patient ages were estimated to be 1.7 years younger than their chronological age before surgery and 8.9 years younger than their chronological age after surgery, with more dramatic results in face and neck lift, upper and lower blepharoplasty, and forehead lift surgeries over face and neck lift surgeries alone [91]. 


\section{Face}

Older patients commonly request "facelifts" to address complaints of facial volume loss and ptosis. Options for facelift include superficial vs. deep-plane traditional rhytidectomy, limited incision approach, and suspension suture lifts. The degree and location of ptosis, extent of volume loss, and extent of malar fat pad descent are important factors to consider. Ideal patients for minimally invasive procedures have minimal skin laxity, good elasticity, no platysmal bands, and no deep cervical creases [92].

The traditional rhytidectomy is divided into superficial and deep plane techniques. Both methods target the superficial muscular aponeurotic system (SMAS), a fibromuscular layer that is deep to the dermis but superficial to the musculoaponeurotic system. It encompasses and distributes force among the facial muscles. Superficial rhytidectomy technique involves either lifting a subcutaneous skin flap or a skin-SMAS flap and redraping the skin temporally and postauricularly, with fixation of the SMAS to the mastoid bone [93]. Deep-plane rhytidectomy is more involved but addresses the problem of malar fat pad descent that occurs with aging and allows for optimal management of the nasolabial fold. A composite flap including the orbicularis oculi muscle and malar fat pad is elevated at the sub-SMAS level and anchored posteriorly [94]. A retrospective study comparing SMAS plication versus deep plane supports SMAS plication in patients under 70 years old and deepplane in patients older than 70 years old. Older patients benefited more so from the deep-plane procedure, compared to the insignificant difference in younger patients [95]. A common complication of these operations is facial nerve paresis.

Limited-incision techniques remove the need for postauricular incision and allow for less noticeable scarring. The limited-incision facelift (LIF) with lateral SMA Sectomy, S-lift, and minimal access cranial suspension lift (MACS-lift) all involve a preauricular incision with superficial dissection and varied levels of SMAS dissection and reapproximation $[96,97]$. These operations differ in incision location and anchoring sites. The S-lift and MACS-lift implement sutures to raise the SMAS and jowls like the LIF with lateral SMASectomy, but do not include platysma resection. S-lift is ideal for younger patients with less ptosis, while MACS-lift is ideal for ptosis of the nasolabial folds and malar fat. The MACS-lift can be simple or extended [98]. The suspension suture lift can be performed with or without the standard rhytidectomy and can be performed closed or open. Ideal patients have poorly defined mandibular border and no significant jowls $[99,100]$. Suture suspension is better for younger patients with only mild to moderate ptosis while barbed sutures are used more for older patients with volume loss [101]. Barbed sutures generally supply less lift but add more volume to the midface [102]. Common complications include asymmetry, dimpling, noticeable scars, granuloma, or transient facial nerve weakness.

The most common complication to rhytidectomy is hematoma formation, with an incidence of up to $15 \%$ [103]. Hematomas can be avoided by maintaining perioperative blood pressure below 150/100 $\mathrm{mmHg}$ and by holding all anticoagulation therapy prior to surgery and obtaining meticulous hemostasis during surgery [104]. Skin flap necrosis is a less common but more severe complication that occurs in up to $3.6 \%$ of procedures [103]. In reconstructive surgery, the most common nerve damaged is the great auricular nerve, leading to numbness of the periauricular area and ear lobe. This can be avoided with careful dissection over the SCM muscle. A more dramatic injury results with damage to the facial nerve. Incidence is 0.1 to $2.6 \%$ and most commonly involves the marginal mandibular and temporal branches. Careful incision planning and conservative flap trimming allows the surgeon to avoid hairline displacement and hypertrophic scars [104].

\section{Eyelids}

Signs of aging in the upper third of the face include brow ptosis and the development of deep forehead skin furrows. Loss of skin elasticity combined with weakened muscle tone accentuates these changes [105]. Surgery aims to reduce forehead wrinkles and raise the brow to give a more youthful appearance. Optimal brow position is at or slightly above the orbital rim and in women, arches about two-thirds along its lateral course. There should be a noticeable distance between the brow and the upper eyelid and a visible eyelid cleft. Options to implement these changes include forehead lifting and blepharoplasty.

Five approaches exist for forehead and brow lifting: direct, coronal, mid-forehead, endoscopic, and trans-blepharoplasty [106]. The direct method allows for a long lasting lift but can leave prominent scarring. The coronal approach provides the best exposure but may result in hair loss, paresthesias, and hematomas. The trichophytic modification of the coronal method creates a less obvious scar line. The midforehead approach is best for deep wrinkles and allows the entire brow to be sculpted while maintaining the hairline. The endoscopic approach is a newer method and conceals scars while minimizing complications, but requires more meticulous technique. According to a recent systematic review, despite increased technical difficulties there is no evidence supporting endoscopic brow surgery over open brow lifting. Open brow-lift is still an excellent choice, especially in patients with short or normal forehead length and deep wrinkles, with results comparable to endoscopic surgery [107]. Forehead and brow lifting method choice depends on patient expectations, level of the hairline, skin type, and surgeon preference but all allows for a more youthful face and pleasing brow position $[108,109]$.

Signs of aging around the eyes specifically include upper lid redundancy, loss of volume with pseudoherniation of lower eyelid fat, and upper eyebrow ptosis. Upper eyelid skin redundancy and orbicularis oculi hypertrophy is corrected by skin and muscle excision [110-112]. Correction of the baggy eyelid can be completed with a cutaneous or transconjunctival approach for lower lid fixation, often combined with lateral retinacular canthopexy. Upper eyebrow ptosis can be managed by forehead lifting, as mentioned above, or with direct browpexy or transblepharoplasty which involve incisions directly above the brow or above the lid. Injection of fat or other fillers can also help increase upper brow volume, helping to create a more youthful appearing eye.

\section{Lips}

The lips are a prominent facial structure that shows specific signs of aging. Aged lips are less full, have blunted Cupid's bow, and an attenuated white roll. Vertical rhytids can also evolve in the upper and lower lids from continued use of the orbicularis muscle [111]. Downward turn of the oral commissures gives a sad or angry "marionette" appearance. Fillers are often used to increase vertical height and increase lip volume, but poor technique or overfilling can result in overt lip projection giving the appearance of a "duck lip." Surgical options aim to raise the upper lip, increase red:white lip ratio, and correct the downward turn of the oral commissures without the use of fillers. Options include vermillion advancement; V-Y mucosal 
advancement, subnasal lift, and corner of the mouth lift [111-113]. Vermillion advancement is performed using an incision along the vermillion border but can result in asymmetry, loss of border definition, and a visible scar, so it is not commonly used. V-Y advancement uses bilateral intraoral incisions and has the benefit of no visible scar but results are unreliable and asymmetry is common. The most commonly used technique is the subnasal lift, in which white lip skin and fascia is resected along vestibule of the nose. Scarring is minimal and asymmetry is less common than the other procedures. The corner of the mouth lift is used to correct the downward turn of oral commissures that may persist through facelift, lip augmentation, or filler use.

\section{Neck}

Multiple reconstructive options exist to redefine the aging neck. Goals of these surgeries include defining the mandibular line, enhancing the prominence of the chin, creating a sharper cervicomental angle (of 105-120 degrees), excising excess submental fat, improving platysmal bands, and defining sternocleidomastoid muscles. The goal of these operations is to create the appearance of a thin, long neck. Suspension sutures can also be used to define the mandibular border. Direct skin excision is an option in men with thick lax submental skin who cannot undergo other procedures. Adjuvant procedures can also be performed for enhanced mental prominence such as chin implant or fillers. The primary surgical options are closed liposuction and platysmaplasty [114]. Closed liposuction offers sharper mandibular and SCM borders and should be performed if the skin is distended less than 15\%; while over $20 \%$ should consider neck lift [114]. A central or submental incision for insertion of tumescence and the liposuction cannula is appropriate. Isolated collections of submental fat may be resected locally instead of through liposuction. As with any cosmetic surgery, common complications include undercorrection, hematomas, and seromas. Platysmaplasty offers contouring of the horizontal neck and formation of a deep cervicomental angle while improving platysmal bands and removing pre- or subplatysmal fat. The procedure is often combined with a cervicofacial rhytidectomy and/or liposuction. A submental incision with dissecting and raising of the muscle, skin, or fat superiorly, creates the appearance of a smoother neck with sharper angles. A postauricular incision can also be used to raise excess skin. Complications include excessive tightening or fat removal, recurrence of bands, and palpable sutures.

\section{Lacking Satisfactory Evidence of Anti-Aging Effect}

This review of the literature showed a lack of high level evidence for age reversal using microdermabrasion, topical stem cells, pentapeptides (KTTKS), collagen creams, and "oxygen" treatments.

\section{Microdermabrasion}

Microdermabrasion is a very popular procedure that can be found in the office of nearly every dermatologist, plastic surgeon, and aesthetician. The American Society for Aesthetic Plastic Surgery alone reported 672,430 procedures performed in 2009 by plastic surgeons and their medical assistants. Microdermabrasion mimics the natural process of exfoliation by mechanically abrading the epidermis with either a diamond tipped wand or a closed-looped system, which blasts fine crystals at the surface of the skin. During this process, only the stratum corneum, which is the outermost layer of the epidermis, is abraded. Between 30-50 passes are required before the stratum corneum is completely removed [115], however standard practice is to perform only 3 passes per session. Thus, complete removal of the stratum corneum is not achieved with microdermabrasion.

Microdermabrasion claims to treat clinical signs of photo aging, but high-level evidence is lacking in the literature. No long-term effects of microdermabrasion on signs of aging, such as collagen deposition or fine lines, have ever been tested. Additionally, no studies have reevaluated skin after regrowth of the stratum corneum. One of the only studies to test the effects of microdermabrasion on photo aged skin was performed by Coimbra and colleagues [116]. In this study, 17 subjects were treated with 8 sessions of microdermabrasion at 1-week intervals after which they were assessed on a 5-point scale by a blinded group of both plastic surgeons $(\mathrm{N}=16)$ and lay people $(\mathrm{N}=14)$. None of the plastic surgeons saw any clinical improvement in fine rhytides, although all evaluators saw mild improvement in hyper pigmentation. Histological analysis of punch biopsies showed increased thickness of the epidermis and increased collagen organization after treatment, which correlates with textural improvement.

There is no evidence that microdermabrasion increases collagen synthesis in the dermis of facial skin unless the coarsest settings and the most aggressive technique are used; however, this aggressive form of treatment also results in more adverse events, such as pinpoint bleeding and bruising $[117,118]$. The moderately low cost per session is appealing, but the consumer should be aware of the minimal effects of each session, requiring multiple sessions to maintain minimal changes. The regimen suggested to patients is at least 6 treatments on a weekly or biweekly basis followed by touch-ups as needed [118]. When performed at moderate settings, this procedure is safe for all skin types with little risk of post inflammatory hyper pigmentation or scarring. Adverse events include mild erythema and pain lasting 1-2 days. Petechiae lasting one week, abrasions, or urticaria are possible following more aggressive sessions. Ocular complications may occur if crystals enter into the eye, and there is a risk of autoinoculation with viruses such as warts, molluscum contagiosum, or herpes simplex [118]. As multiple sessions are required, the therapy becomes expensive with no evidence that effects are maintained over time.

\section{Topical Stem Cells}

While adipose derived stem cells may have an effect on the signs of aging when injected subcutaneously in animal models [119], there is no evidence of a benefit following topical application. It is unlikely that moisturizing creams infused with stem cells result in any added benefit to skin.

\section{Peptides: Topical Growth Factors, Pentapeptide (KTTKS) and Collagen Creams}

Growth factors have been reported to increase collagen production in several small, product specific, corporately funded studies lacking a control group [120,121]. It is unclear whether the results of these studies were due to the growth factors alone, the vehicle, or another confounding variable. For example, some creams contained additional active ingredients such as vitamin $\mathrm{C}$ (L-ascorbic acid), thus the benefit of the growth factors alone is uncertain. The results of these studies have not been largely reproduced and an explanation for the mechanism of action is still unclear. Growth factors, cytokines, and other proteins are large hydrophilic molecules on a scale of 15,000 Daltons (Da) or larger [120]. Large hydrophilic molecules larger than 500DA are known to have very low penetration through the stratum 
Page 9 of 12

corneum [122,123]. Therefore treatments involving large proteins would logically be unable to penetrate the skin to effect change unless the skin was compromised in some way. Because growth factors, such as those derived from fetal fibroblast, have proven to be beneficial for cases in which the outer epidermal layer is lost, such as wound healing and the treatment of burns [124-126], there may be some benefit to using these creams in combination with procedures that interrupt the outer layers of the skin, such as chemical peel or ablative laser therapy. Combination therapy has not yet been studied.

Pentapeptide or KTTKS, a 5 amino acid chain of Lysine, Threonine, Lysine, and Threonine has been popularized recently, but similar to growth factors, this molecule is hydrophilic and has a molecular weight of $563 \mathrm{Da}$ [127], which is too large to penetrate the skin. Penetration enhancers may be a way to overcome this barrier, but the addition of bulky side groups may actually make penetration more difficult. The literature is lacking in well controlled studies of this compound, but one double-blind, placebo-controlled, split-faced study did show a small but significant improvement upon clinical assessment of fine lines and wrinkles with KTTKS cream over a placebo cream after 12 weeks of use [128]. However, because this molecule is not be able to penetrate the skin, the effect may be due to some ability to superficially fill in lines and smooth the skin's surface without actually penetrating the epidermis to stimulate collagen or elastin production within the layers of the skin.

For this same reason, topical collagen creams are theoretically ineffective because they are unable to penetrate the outer layer of the epidermis. Thus, topical collagen creams are unable to build upon the skin's own collagen networks.

\section{Oxygen}

Some products on the market claim to impart oxygen to the skin. Often, there is no specific ingredient that is identified as the oxygenbearing compound or that is attributed with the ability to convert toxins into oxygen. This seems to be pure marketing strategy, as no studies have investigated the isolated effects of topical oxygen creams.

\section{Conclusion}

Aging is an ongoing intrinsic process, hastened by external factors such as UV exposure and toxins. Signs of aging include a loss of facial volume, thinning of the epidermis and dermis, dyspigementation, elastosis, and both fine and course wrinkles. Just as factors exist which accelerate aging, interventions also exist to counteract or even reverse the effects of aging. Among the most recommended and clinically effective minimally invasive interventions are sunscreens, antioxidants, retinoids, chemical peels, botulinum toxin, ablative fractional photothermolysis, and photodynamic therapy. Study of other novel therapies such as topical growth factors and peptides, although promising has yet to achieve an adequate level of evidence to substantiate claims or to clarify a mechanism of action when applied topically to intact skin. While popular, micro-dermabrasion provides only fleeting benefits and minimal age-reversal in the long term. Surgical intervention is now often supplemented with fat grafting and neuromodulators. As new anti-aging therapies are developed at an accelerated pace, critical appraisal of clinically proven effects can help guide selection of the most appropriate therapies for each individual's anti-aging needs and desired outcome.

\section{Conflicts of Interest}

The authors have no financial interest in any product or piece of equipment discussed in this article.

\section{References}

1. Phillips B, Ball C, Sackett D, Badenoch D, Straus S, et al. (2009) Oxford centre for evidence-based medicine-levels of evidence.

2. Puizina-Ivic N, Miric L, Carija A, Karlica D, Marasovic D (2010) Modern approach to topical treatment of aging skin. Coll Antropol 34: 1145-1153.

3. Dulguerov N, D'Souza A (2011) Update on treatment rationale and options for the ageing face. Curr Opin Otolaryngol Head Neck Surg 19: 269-275.

4. Kahn DM, Shaw RB (2010) Overview of current thoughts on facial volume and aging. Facial Plast Surg 26: 350-355.

5. Muzaffar AR, Mendelson BC, Adams WP Jr (2002) Surgical anatomy of the ligamentous attachments of the lower lid and lateral canthus. Plast Reconstr Surg 110: 873-884.

6. Yousif NJ, Gosain A, Sanger JR, Larson DL, Matloub HS (1994) The nasolabial fold: a photogrammetric analysis. Plast Reconstr Surg 93:70-77.

7. Pessa JE (2000) An algorithm of facial aging: verification of Lambros's theory by three-dimensional stereolithography, with reference to the pathogenesis of midfacial aging, scleral show, and the lateral suborbital trough deformity. Plast Reconstr Surg 106:479-488.

8. Okada HC, Alleyne B, Varghai K, Kinder K, Guyuron B (2013) Facial changes caused by smoking: a comparison between smoking and nonsmoking identical twins. Plast Reconstr Surg 132: 1085-1092.

9. Narins RS, Carruthers J, Flynn TC, Geister TL, Gortelmeyer R, et al. (2012) Validated assessment scales for the lower face. Dermatol Surg 38: 333-342.

10. Carruthers J, Flynn TC, Geister TL, Gortelmeyer R, Hardas B, et al. (2012) Validated assessment scales for the mid face. Dermatol Surg 38: 320-332.

11. Flynn TC, Carruthers A, Carruthers J, Geister TL, Gortelmeyer R, et al. (2012) Validated assessment scales for the upper face. Dermatol Surg 38: 309-319.

12. Rzany B, Carruthers A, Carruthers J, Flynn TC, Geister TL, et al. (2012) Validated composite assessment scales for the global face. Dermatol Surg 38(2 Spec No.

13. Alexiades-Armenakas M (2006) A quantitative and comprehensive grading scale for rhytides, laxity, and photoaging. J Drugs Dermatol 5: 808-809.

14. Battistutta D, Pandeya N, Strutton GM, Fourtanier A, Tison S, et al. (2006) Skin surface topography grading is a valid measure of skin photoaging. Photodermatol Photoimmunol Photomed 22: 39-45.

15. Hughes MC, Strutton GM, Fourtanier A, Green AC (2012) Validation of skin surface microtopography as a measure of skin photoaging in a subtropical population aged 40 and over. Photodermatol Photoimmunol Photomed 28: 153-158.

16. Sambandan DR, Ratner D (2011) Sunscreens: an overview and update. J Am Acad Dermatol 64: 748-758.

17. Stern RS (2004) Clinical practice. Treatment of photoaging. N Engl J Med 350: 1526-1534.

18. Hughes MC, Williams GM, Baker P, Green AC (2013) Sunscreen and prevention of skin aging: a randomized trial. Ann Intern Med 158: 781-790.

19. Kricker A, Armstrong BK, English DR, Heenan PJ (1991) Pigmentary and cutaneous risk factors for non-melanocytic skin cancer--a casecontrol study. Int J Cancer 48: 650-662.

20. Holman CD, Armstrong BK, Evans PR, Lumsden GJ, Dallimore KJ, et al. (1984) Relationship of solar keratosis and history of skin cancer to objective measures of actinic skin damage. Br J Dermatol 110: 129-138. 
21. Green A, Williams G, Neale R, Hart V, Leslie D, et al. (1999) Daily sunscreen application and betacarotene supplementation in prevention of basal-cell and squamous-cell carcinomas of the skin: a randomised controlled trial. Lancet 354: 723-729.

22. Chen L, Hu JY, Wang SQ (2012) The role of antioxidants in photoprotection: a critical review. J Am Acad Dermatol 67: 1013-1024.

23. Kawaguchi Y, Tanaka H, Okada T, Konishi H, Takahashi M, et al. (1997) Effect of reactive oxygen species on the elastin mRNA expression in cultured human dermal fibroblasts. Free Radic Biol Med 23: 162-165.

24. Bernstein EF (2002) Reactive oxygen species activate the human elastin promoter in a transgenic model of cutaneous photoaging. Dermatol Surg 28: $132-135$.

25. Uitto J (2008) The role of elastin and collagen in cutaneous aging: intrinsic aging versus photoexposure. J Drugs Dermatol 7: s12-16.

26. Rhie G, Shin MH, Seo JY, Choi WW, Cho KH, et al. (2001) Aging- and photoaging-dependent changes of enzymic and nonenzymic antioxidants in the epidermis and dermis of human skin in vivo. J Invest Dermatol 117: 1212-1217

27. Lin JY, Selim MA, Shea CR, Grichnik JM, Omar MM, et al. (2003) UV photoprotection by combination topical antioxidants vitamin $\mathrm{C}$ and vitamin E. J Am Acad Dermatol 48: 866-874.

28. Campos PM, Gonçalves GM, Gaspar LR (2008) In vitro antioxidant activity and in vivo efficacy of topical formulations containing vitamin $\mathrm{C}$ and its derivatives studied by non-invasive methods. Skin Res Technol 14: 376-380.

29. Nayama S, Takehana M, Kanke M, Itoh S, Ogata E, et al. (1999) Protective effects of sodium-L-ascorbyl-2 phosphate on the development of UVB-induced damage in cultured mouse skin. Biol Pharm Bull 22: 1301-1305.

30. Pinnell SR, Yang H, Omar M, Monteiro-Riviere N, DeBuys HV, et al (2001) Topical L-ascorbic acid: percutaneous absorption studies. Dermatol Surg 27: 137-142.

31. Matsui MS, Hsia A, Miller JD, Hanneman K, Scull H, et al. (2009) Nonsunscreen photoprotection: antioxidants add value to a sunscreen. Investig Dermatol Symp Proc 14: 56-59.

32. Wu Y, Matsui MS, Chen JZ, Jin X, Shu CM, et al. (2011) Antioxidants add protection to a broad-spectrum sunscreen. Clin Exp Dermatol 36: 178-187.

33. Wang SQ, Stanfield JW, Osterwalder U (2008) In vitro assessments of UVA protection by popular sunscreens available in the United States. J Am Acad Dermatol 59: 934-942.

34. Palmer DM, Kitchin JS (2010) A double-blind, randomized, controlled clinical trial evaluating the efficacy and tolerance of a novel phenolic antioxidant skin care system containing coffea arabica and concentrated fruit and vegetable extracts. J Drugs Dermatol 9: 1480-1487.

35. Kang S, Voorhees JJ (1998) Photoaging therapy with topical tretinoin: an evidence-based analysis. J Am Acad Dermatol 39: S55-61.

36. Varani J, Fisher GJ, Kang S, Voorhees JJ (1998) Molecular mechanisms of intrinsic skin aging and retinoid-induced repair and reversal. J Investig Dermatol Symp Proc 3: 57-60.

37. Varani J, Perone P, Griffiths CEM, Inman DR, Filgiel SE, et al. (1994) All trans retinoic acid (RA) stimulates events in organ-cultured human skin that underlie repair: Adult skin from sun-protected and sun exposed sites responds in an identical manner to RA while neonatal foreskin responds differently. J Clin Invest 94: 1747-1756.

38. Varanji J, Warner RL, Gharaee-Kermani M, Phan SH, Kang S, et al. (2000) Vitamin A antagonizes decreased cell growth and elevated collagen-degrading matrix metalloproteinases and stimulates collagen accumulation in naturally aged human skin. J Invest Dermatol 114: 480-486.

39. Kligman AM (2003) Topical retinoic acid for photo aging: conceptions and misperceptions. Cosmet Dermatol 16: S3-S6.

40. Pierard-Franchimont C, Castelli D, Van Cromphaut I, Bertin C, Ries G, et al. (1998) Tensile properties and contours of aging facial skin. A controlled double-blind comparative study of the effects of retinol, melibiose-lactose and their association. Skin Res Technol 4: 237-243.

41. Kafi R, Kwak HS, Schumacher WE, Cho S, Hanft VN, et al. (2007) Improvement of naturally aged skin with vitamin A (retinol). Arch Dermatol 143: 606-612.

42. Lee MS, Lee KH, Sin HS, Um SJ, Kim JW, et al. (2006) A newly synthesized photostable retinol derivative (retinyl $\mathrm{N}$-formyl aspartamate) for photodamaged skin: profilometric evaluation of 24-week study. J Am Acad Dermatol 55: 220-224.

43. Griffiths CE, Kang S, Ellis CN, Kim KJ, Finkel LJ, et al. (1995) Two concentrations of topical tretinoin (retinoic acid) cause similar improvement of photoaging but different degrees of irritation. A doubleblind, vehicle-controlled comparison of $0.1 \%$ and $0.025 \%$ tretinoin creams. Arch Dermatol 131: 1037-1044.

44. Nyirady J, Bergfeld W, Ellis C, Levine N, Savin R, et al. (2001) Tretinoin cream $0.02 \%$ for the treatment of photodamaged facial skin: a review of 2 double-blind clinical studies. Cutis 68: 135-142.

45. Mukherjee S, Date A, Patravale V, Korting HC, Roeder A, et al. (2006) Retinoids in the treatment of skin aging: an overview of clinical efficacy and safety. Clin Interv Aging 1: 327-348.

46. Leyden JJ, Grove GL, Grove MJ, Thorne EG, Lufrano L (1989) Treatment of photodamaged facial skin with topical tretinoin. J Am Acad Dermatol 21: 638-644.

47. Weinstein GD, Nigra TP, Pochi PE, Savin RC, Allan A, et al. (1991) Topical tretinoin for treatment of photodamaged skin. A multicenter study. Arch Dermatol 127: 659-665.

48. Olsen EA, Katz HI, Levine N, Shupack J, Billys MM, et al. (1992) Tretinoin emollient cream: a new therapy for photodamaged skin. J Am Acad Dermatol 26: 215-224.

49. Kang S, Bergfeld W, Gottlieb AB, Hickman J, Humeniuk J, et al. (2005) Long-term efficacy and safety of tretinoin emollient cream $0.05 \%$ in the treatment of photodamaged facial skin: a two-year, randomized, placebocontrolled trial. Am J Clin Dermatol 6: 245-253.

50. Ellis CN, Weiss JS, Hamilton TA, Headington JT, Zelickson AS, et al. (1990) Sustained improvement with prolonged topical tretinoin (retinoic acid) for photoaged skin. J Am Acad Dermatol 23: 629-637.

51. Green LJ, McCormick A, Weinstein GD (1993) Photoaging and the skin. The effects of tretinoin. Dermatol Clin 11: 97-105.

52. Elder JT, Kaplan A, Cromie MA, Kang S, Voorhees JJ (1996) Retinoid induction of CRABP II mRNA in human dermal fibroblasts: use as a retinoid bioassay. J Invest Dermatol 106: 517-521.

53. Varani J, Zeigler M, Dame MK, Kang S, Fisher GJ, et al. (2001) Heparinbinding epidermal-growth-factor-like growth factor activation of keratinocyte ErbB receptors Mediates epidermal hyperplasia, a prominent side-effect of retinoid therapy. J Invest Dermatol 117: 1335-1341.

54. Baumann L (2007) Skin ageing and its treatment. J Pathol 211: 241-251.

55. Kang S, Duell EA, Fisher GJ, Datta SC, Wang ZQ, et al. (1995) Application of retinol to human skin in vivo induces epidermal hyperplasia and cellular retinoid binding proteins characteristic of retinoic acid but without measurable retinoic acid levels or irritation. J Invest Dermatol 105: 549-556.

56. Kurlandsky SB, Xiao JH, Duell EA, Voorhees JJ, Fisher GJ (1994) Biological activity of all-trans retinol requires metabolic conversion to all-trans retinoic acid and is mediated through activation of nuclear retinoid receptors in human keratinocytes. J Biol Chem 269: 32821-32827.

57. Duell EA, Kang S, Voorhees JJ (1997) Unoccluded retinol penetrates human skin in vivo more effectively than unoccluded retinyl palmitate or retinoic acid. J Invest Dermatol 109: 301-305.

58. Ho ET, Trookman NS, Sperber BR, Rizer RL, Spindler R, et al. (2012) A randomized, double-blind, controlled comparative trial of the anti-aging properties of non-prescription tri-retinol $1.1 \%$ vs. prescription tretinoin 0.025\%. J Drugs Dermatol 11: 64-69. 
59. Mangat DS, Tansavatdi K, Garlich P (2011) Current chemical peels and other resurfacing techniques. Facial Plast Surg 27: 35-49.

60. Rendon MI, Berson DS, Cohen JL, Roberts WE, Starker I, et al. (2010) Evidence and considerations in the application of chemical peels in skin disorders and aesthetic resurfacing. J Clin Aesthet Dermatol 3: 32-43.

61. Oresajo C, Yatskayer M, Hansenne I (2008) Clinical tolerance and efficacy of capryloyl salicylic acid peel compared to a glycolic acid peel in subjects with fine lines/wrinkles and hyperpigmented skin. J Cosmet Dermatol 7: 259-262.

62. Langsdon PR, Rodwell DW 3rd, Velargo PA, Langsdon CH, Guydon A (2012) Latest chemical peel innovations. Facial Plast Surg Clin North Am 20: 119-123, v.

63. Tse Y, Ostad A, Lee HS, Levine VJ, Koenig K, et al. (1996) A clinical and histologic evaluation of two medium-depth peels. Glycolic acid versus Jessner's trichloroacetic acid. Dermatol Surg 22: 781-786.

64. Sweeney LM, Kirman CR, Gargas ML, Dugard PH (2009) Contribution of trichloroacetic acid to liver tumors observed in perchloroethylene (perc)-exposed mice. Toxicology 260: 77-83.

65. Varshney M, Chandra A, Chauhan LK, Goel SK (2013) In vitro cytogenetic assessment of trichloroacetic acid in human peripheral blood lymphocytes. Environ Sci Pollut Res Int 21: 843-850.

66. NTP (National Toxicology Program) (1986) Toxicology and carcinogenesis studies of tetrachloroethylene (perchloroethylene) in F344/N rats and B6C3F1 mice. U.S Department of Health and Human Services, Technical Report Series 311: 1-190.

67. National Toxicology Program (2011) NTP 12th Report on Carcinogens. Rep Carcinog 12: iii-499.

68. Flynn TC (2012) Advances in the use of botulinum neurotoxins in facial esthetics. J Cosmet Dermatol 11: 42-50.

69. Lorenc PZ, Kenkel JM, Fagien S, Hirmand H, Nestor MS, et al. (2012) A Review of OnabotulinumtoxinA (Botox). Aesthetic Surgery Journal 33: 85-125.

70. Glasgold RA, Lam SM, Glasgold MJ (2008) Facial fat grafting: the new paradigm. Arch Facial Plast Surg 10: 417-418.

71. Ciuci PM, Obagi S (2008) Rejuvenation of the periorbital complex with autologous fat transfer: current therapy. J Oral Maxillofac Surg 66: 1686-1693.

72. Glashofer M, Lawrence N (2006) Fat transplantation for treatment of the senescent face. Dermatol Ther 19: 169-176.

73. Tabit CJ, Slack GC, Fan K, Wan DC, Bradley JP (2012) Fat Grafting Versus Adipose- Derived Stem Cell Therapy: Distinguishing Indications, Techniques, and Outcomes. Aesthetic Plast Surg 36: 704-713.

74. Guijarro-Martínez R, Miragall Alba L, Marqués Mateo M, Puche Torres M, Pascual Gil JV (2011) Autologous fat transfer to the craniomaxillofacial region: updates and controversies. J Craniomaxillofac Surg 39: $359-363$

75. Fontdevila J, Serra-Renom JM, Raigosa M, Berenguer J, Guisantes E, et al. (2008) Assessing the long-term viability of facial fat grafts: an objective measure using computed tomography. Aesthet Surg J 28: 380-386.

76. Sterodimas A, de Faria J, Nicaretta B, Boriani F (2011) Autologous fat transplantation versus adipose-derived stem cell-enriched lipografts: a study. Aesthet Surg J 31: 682-693.

77. Latowsky BC, Abbasi N, Dover JS, Arndt KA, Kaminer MS, et al. (2012) A randomized, controlled trial of four ablative fractionated lasers for photoaging: a quadrant study. Dermatol Surg 38: 1477-1489.

78. Tierney EP, Hanke CW (2011) Fractionated carbon dioxide laser treatment of photoaging: prospective study in 45 patients and review of the literature. Dermatol Surg 37: 1279-1290.

79. Alexiades-Armenakas MR, Dover JS, Arndt KA (2008) The spectrum of laser skin resurfacing: nonablative, fractional, and ablative laser resurfacing. J Am Acad Dermatol 58: 719-737.

80. Ortiz AE, Tremaine AM, Zachary CB (2010) Long-term efficacy of a fractional resurfacing device. Lasers Surg Med 42: 168-170.
81. Tierney EP, Hanke CW (2009) Ablative fractionated CO2, laser resurfacing for the neck: prospective study and review of the literature. J Drugs Dermatol 8: 723-731.

82. Kotlus BS (2010) Dual-depth fractional carbon dioxide laser resurfacing for periocular rhytidosis. Dermatol Surg 36: 623-628.

83. Karrer S, Kohl E, Feise K, Hiepe-Wegener D, Lischner S, et al. (2012) Photodynamic therapy for skin rejuvenation: review and summary of the literature - results of a consensus conference of an expert group for aesthetic photodynamic therapy. JDDG 11: 137-148.

84. Gaulloer JM, Berg K, Peng Q (1997) Use of 5-Aminolevulinic acid esters to improve photodynamic therapy on cells in culture ALA to improve PDT on cells in culture. Cancer Res 57: 1481-1486.

85. Kennedy JC, Pottier RH, Pross DC (1990) Photodynamic therapy with endogenous protoporphyrin IX: basic principles and present clinical experience. J Photochem Photobiol B 6: 143-148.

86. Kosaka S, Yasumoto M, Akilov OE, Hasan T, Kawana S (2010) Comparative split-face study of 5-aminolevulinic acid photodynamic therapy with intense pulsed light for photorejuvenation of Asian skin. J Dermatol 37: 1005-1010.

87. Dover JS, Bhatia AC, Stewart B, Arndt KA (2005) Topical 5aminolevulinic acid combined with intense pulsed light in the treatment of photoaging. Arch Dermatol 141: 1247-1252.

88. Issa MC, Pineiro-Maceira J, Vieira MT, Olej B, Mandarim-De-Lacerda CA, et al. (2010) Photorejuvenation with topical methyl aminolevulinate and red light: a randomized, prospective, clinical, histopathologic, and morphometric study. Dermatol Surg 36: 39-48.

89. Karrer S, Kohl E, Feise K, Hiepe-Wegener D, Lischner S, et al. (2012) Photodynamic therapy for skin rejuvenation: review and summary of the literature--results of a consensus conference of an expert group for aesthetic photodynamic therapy. J Dtsch Dermatol Ges 11: 137-148.

90. Haedersdal M, Katsnelson J, Sakamoto FH, Farinelli WA, Doukas AG, et al. (2011) Enhanced uptake and photoactivation of topical methyl aminolevulinate after fractional Co2 laser pretreatment. Lasers Surg Med 43: 804-813.

91. Chauhan N, Warner JP, Adamson PA (2012) Perceived age change after aesthetic facial surgical procedures quantifying outcomes of aging face surgery. Arch Facial Plast Surg 14: 258-262.

92. Carniol PJ, Ganc DT (2007) Is there an ideal facelift procedure? Curr Opin Otolaryngol Head Neck Surg 15: 244-252.

93. Ivy EJ, Lorenc ZP, Aston SJ (1996) Is there a difference? A prospective study comparing lateral and standard SMAS face lifts with extended SMAS and composite rhytidectomies. Plast Reconstr Surg 98: 1135-1143.

94. Hamra ST (1990) The deep-plane rhytidectomy. Plast Reconstr Surg 86: 53-61.

95. Becker FF, Bassichis BA (2004) Deep-plane face-lift vs superficial musculoaponeurotic system plication face-lift: a comparative study. Arch Facial Plast Surg 6: 8-13.

96. Baker DC (2001) Minimal incision rhytidectomy (short scar face lift) with lateral SMASectomy: evolution and application. Aesthet Surg J 21: 14-26.

97. Tonnard PL, Verpaele A, Gaia S (2005) Optimising results from minimal access cranial suspension lifting (MACS-lift). Aesthetic Plast Surg 29: 213-220.

98. Verpaele A, Tonnard P, Gaia S, Guerao FP, Pirayesh A (2007) The third suture in MACS-lifting: making midface-lifting simple and safe. J Plast Reconstr Aesthet Surg 60: 1287-1295.

99. Sasaki GH, Cohen AT (2002) Meloplication of the malar fat pads by percutaneous cable-suture technique for midface rejuvenation: outcome study (392 cases, 6 years' experience). Plast Reconstr Surg 110: 635-654.

100. Keller GS, Namazie A, Blackwell K, Rawnsley J, Khan S (2002) Elevation of the malar fat pad with a percutaneous technique. Arch Facial Plast Surg 4: 20-25.

101. Khawaja HA, Hernandez-Pérez E (2005) Transcutaneous face-lift. Dermatol Surg 31: 453-457. 
Citation: Salgado CJ, LaSenna CE, Cissel R, Li X, Gordon CE, et al. (2014) Discovering the 21st Century Fountain of Youth: A Discussion of the Effectiveness of Popular Anti-Aging Therapies. Anaplastology 3: 127. doi:10.4172/2161-1173.1000127

Page 12 of 12

102. Lee S, Isse N (2005) Barbed polypropylene sutures for midface elevation: early results. Arch Facial Plast Surg 7: 55-61.

103. Griffin JE, Jo C (2007) Complications after superficial plane cervicofacial rhytidectomy: a retrospective analysis of 178 consecutive facelifts and review of the literature. J Oral Maxillofac Surg 65: 2227-2234.

104. Rodriguez-Bruno K, Papel ID (2011) Rhytidectomy: principles and practice emphasizing safety. Facial Plast Surg 27: 98-111.

105. Presti P, Yalamanchili H, Honrado CP (2006) Rejuvenation of the aging upper third of the face. Facial Plast Surg 22: 91-96.

106. Angelos PC, Stallworth CL, Wang TD (2011) Forehead lifting: state of the art. Facial Plast Surg 27: 50-57.

107. Graham DW, Heller J, Kurkjian TJ, Schaub TS, Rohrich RJ (2011) Brow lift in facial rejuvenation: a systematic literature review of open versus endoscopic techniques. Plast Reconstr Surg 128: 335e-341e.

108. Ross AT, Neal JG (2006) Rejuvenation of the aging eyelid. Facial Plast Surg 22: 97-104.

109. Pastorek NJ (2002) Blepharoplasty update. Facial Plast Surg Clin North Am 10: 23-27, vii.

110. Trussler AP, Rohrich RJ (2008) MOC-PSSM CME article: Blepharoplasty. Plast Reconstr Surg 121: 1-10.

111. Ali MJ, Ende K, Maas CS (2007) Perioral rejuvenation and lip augmentation. Facial Plast Surg Clin North Am 15: 491-500, vii.

112. Segall L, Ellis DA (2007) Therapeutic options for lip augmentation. Facial Plast Surg Clin North Am 15: 485-490.

113. Perkins SW (2007) The corner of the mouth lift and management of the oral commissure grooves. Facial Plast Surg Clin North Am 15: 471-476.

114. Patel BC (2006) Aesthetic surgery of the aging neck: options and techniques. Orbit 25: 327-356.

115. Gill HS, Andrews SN, Sakthivel SK, Fedanov A, Williams IR, et al. (2009) Selective removal of stratum corneum by microdermabrasion to increase skin permeability. Eur J Pharm Sci 38: 95-103.

116. Coimbra M, Rohrich RJ, Chao J, Brown SA (2004) A prospective controlled assessment of microdermabrasion for damaged skin and fine rhytides. Plast Reconstr Surg 113: 1438-1443.
117. Karimipour DJ, Karimipour G, Orringer JS (2010) Microdermabrasion: an evidence-based review. Plast Reconstr Surg 125: 372-377.

118. Karimipour DJ, Rittié L, Hammerberg C, Min VK, Voorhees JJ, et al. (2009) Molecular analysis of aggressive microdermabrasion in photoaged skin. Arch Dermatol 145: 1114-1122.

119. Kim WS, Park BS, Sung JH (2009) Protective role of adipose-derived stem cells and their soluble factors in photoaging. Arch Dermatol Res 301: 329-336.

120. Mehta RC, Fitzpatrick RE (2007) Endogenous growth factors as cosmeceuticals. Dermatol Ther 20: 350-359.

121. Fitzpatrick RE, Rostan EF (2003) Reversal of photodamage with topical growth factors: a pilot study. J Cosmet Laser Ther 5: 25-34.

122. Bos JD, Meinardi MM (2000) The 500 Dalton rule for the skin penetration of chemical compounds and drugs. Exp Dermatol 9: 165-169.

123. Jakasa I, Verberk MM, Esposito M, Bos JD, Kezic S (2007) Altered penetration of polyethylene glycols into uninvolved skin of atopic dermatitis patients. J Invest Dermatol 127: 129-134.

124. Naughton G, Mansbridge J, Gentzkow G (1997) A metabolically active human dermal replacement for the treatment of diabetic foot ulcers. Artif Organs 21: 1203-1210.

125. Hohlfeld J, de Buys Roessingh A, Hirt-Burri N, Chaubert P, Gerber S, et al. (2005) Tissue engineered fetal skin constructs for paediatric burns. Lancet 366: 840-842.

126. Cass DL, Meuli M, Adzick NS (1997) Scar wars: implications of fetal wound healing for the pediatric burn patient. Pediatr Surg Int 12 : 484-489.

127. Abu Samah NH, Heard CM (2011) Topically applied KTTKS: a review. Int J Cosmet Sci 33: 483-490.

128. Robinson LR, Fitzgerald NC, Doughty DG, Dawes NC, Berge CA, et al. (2005) Topical palmitoyl pentapeptide provides improvement in photoaged human facial skin. Int J Cosmet Sci 27: 155-160. 